\title{
O PROJETO DE GRADUAÇÃO E A PRODUÇÃO CIENTÍFICA: ESTUDO DE CASO DO CURSO DE OCEANOGRAFIA DO CTTMAR-UNIVALI
}

\author{
PROENÇA, L.A.O.; FONSECA, R.S. \& M.I.F. DOS SANTOS \\ Centro de Ciências Tecnológicas da Terra e do mar, CTTMar, Univali. Rua Uruguai, \\ 458, Centro Itajaí, SC \\ proenca@cttma.univali.br
}

RESUMO

\begin{abstract}
O presente trabalho visa caracterizar os Projetos de Graduação do Curso de Oceanografia da UNIVALI enquanto meio de produção científica. O levantamento foi feito com entrevistas aos orientadores dos trabalhos, tendo como base a produção bibliográfica disponível na Plataforma Lattes, do CNPq. Em média, cada projeto teve uma produção igual a 1,5, nas categorias: artigos completos em periódicos, capítulos de livros, trabalhos completos em anais e resumos apresentados em congressos, excluindo-se participações em seminários de iniciação científica. Resumos em congresso foi a categoria com maior contribuição. Considerando a produção dos orientadores, os PG representam cerca de $10 \%$ do total. Os resultados indicam que, além de atender aos objetivos da disciplina, os projetos de graduação são um importante meio de iniciação científica para o aluno e colaboram para a produção total dos orientadores.
\end{abstract}

Palavras chave: monografia, dissertação, conclusão de curso, produção científica, oceanografia.

\section{SCIENCE PRODUCTION AND THE UNDERGRADUATE PROJECT: A CASE STUDY OF THE OCEANOGRAPHY AT CTTMAR-UNIVALI.}

\begin{abstract}
This paper aims to investigate and quantify the science generated by the undergraduate project carried out by Oceanography students from UNIVALI. The data was obtained by interviews with the projects supervisers and search using the CNPq LATES bibliographic production data base. In general, each undergraduate project produced 1.5 scientific contribution, within journal paper, book chapter, proceedings full paper or proceedings abstract categories. This later category was the one that produced the greater contribution. About $10 \%$ of the scientific production of the superviser where related to the projects. The results shows that apart from the their objectives, which are driven to the student formation, the undergraduate project also contribute to the science production of the Oceanography Course.
\end{abstract}

Key words: undergraduate project, scientific production, oceanography, undergraduate monograph.

\section{INTRODUÇÃO}

Desde sua implantação, no ano de 1992, a disciplina de Projeto de Graduação (PG) faz parte da matriz curricular do Curso de Oceanografia da Univali e se constitui numa importante atividade profissionalizante. A disciplina tem como objetivos: a) complementar atividade de aprendizagem teórica e prática nos diferentes campos de atuação profissional, na Univali ou em outras instituições de ensino e pesquisa e em empresas públicas e privadas; 
b) desenvolver atitudes e hábitos profissionais, bem como, adquirir, exercitar e aprimorar conhecimentos técnicos e/ou científicos no campo de atividade do projeto; c) estimular a especialização em um campo de atividade específica; d) promover a integração entre o acadêmico formando e o mercado de trabalho. $\mathrm{Na}$ maneira como a disciplina esta montada, estes objetivos são alcançados com o desenvolvimento de um projeto elaborado pelo aluno sob orientação de um profissional qualificado. O projeto se desenvolve durante dois semestres acadêmicos, aproximadamente nove meses. A disciplina conta com certos prérequisitos que visam assegurar que $o$ aluno tenha já adquirido a maior parte dos conhecimentos básicos e profissionalizantes que o curso de oceanografia oferece. Assim, geralmente, os alunos que cursam a disciplina estão em vias da conclusão do Curso (ProEn, 2003, www.cttmar.univali.br/ graduacao/).

No Curso de Oceanografia-UNIVALI fazem parte do $P G$ o aluno, seu orientador, eventualmente um co-orientador e o professor responsável pela disciplina. Este último, entre outros afazeres, coordena as atividades, estabelece o calendário da disciplina e atua como mediador entre o aluno e seu orientador. O PG pode ser dividido em quatro etapas básicas: elaboração do projeto, desenvolvimento do trabalho (trabalho de conclusão de curso ou graduação), redação de uma monografia e sua avaliação. Na primeira etapa, o aluno após estabelecer contato com um orientador, elege um tema de trabalho e elabora um projeto a ser desenvolvido em dois semestres letivos. O projeto orientado é produzido pelo aluno e encaminhado a dois revisores. Após a revisão, os projeto é aprovado e está pronto para ser executado. $\mathrm{O}$ projeto pode ser de pesquisa ou de estágio. No primeiro, o aluno atinge os objetivos da disciplina por meio de investigação, enquanto no segundo, por meio do treinamento orientado (http:// www.cttmar.univali.br/ graduacao/).

Na segunda etapa, o aluno desenvolve o trabalho prático, sempre sob orientação. $O$ trabalho prático é dimensionado de forma a possibilitar sua conclusão dentro dos prazos estabelecidos. Após a fase de obtenção de dados, seu tratamento e análise, o aluno desenvolve uma monografia, seguindo normas da redação científica. Após sua redação, o trabalho é exposto nas formas orais e escrita a uma banca para sua avaliação. A banca, formada por professores do curso ou convidados, avalia o trabalho levando-se em conta os objetivos da disciplina.

Embora seja desejável, nem sempre um Projeto de Graduação produz um material científico no senso estrito. Muitas vezes o aluno obtém habilidades profissionais, como o manejo de um determinado equipamento, ou uma técnica de tratamento de dados, sem que exista produção científica. Mesmo na avaliação do TCC pela banca, o aluno pode demonstrar um grande aprendizado e ser aprovado, mesmo no caso que o trabalho apresente falhas quanto ao método ou amostragem, por exemplo.

Muitas vezes os trabalhos contém material que necessita ser mais elaborado, revisado e discutido para assim ser apresentando como uma contribuição científica. Porém, em certos casos, dado suas características, o rigor em seu desenvolvimento, dedicação e maturidade acadêmica do aluno, entre outros, o projeto representa uma contribuição genuína à ciência. O presente trabalho visa descrever e avaliar produção científica baseada nos Projetos de Graduação do Curso de Oceanografia da Univali.

\section{MATERIAL E MÉTODOS}

Para esta pesquisa foram considerados 119 PGs desenvolvidos desde a criação do Curso em 1992 até o primeiro semestre de 2002. A lista dos trabalhos analisados está no anexo 1. Optou-se para elaborar o inventário das publicações originadas dos $\mathrm{PGs}$ por meio de consulta aos orientadores, tendo como base a produção científica listada no Currículo Lattes (Plataforma Lattes, CNPq). Os CV-Lattes foram 
obtidos pela rede internet de computadores por consulta direta no site http://lattes.cnpq.br/. De posse do CV-Lattes atualizado, os orientadores foram entrevistados e solicitados e identificar, entre sua produção, quais trabalhos foram fundamentados em PGs orientados no Curso de Oceanografia. Nesta pesquisa, realizada em março de 2003, teve-se como pressuposto, que a produção gerada pelo PG contou com o orientador como autor ou co-autor. Uma vez identificada uma publicação na qual houve a contribuição de um PG, foi solicitado ao entrevistado que classificasse esta contribuição conforme as categorias abaixo apresentadas. Desta maneira, a separação entre as categorias partiu dos próprios orientadores: Trabalhos totalmente baseados em PGs. (100\% de contribuição); Trabalhos baseados em sua maior parte em PGs (contribuição maior que $50 \%$ ); Trabalhos baseados em pequena parte em PGs ( > 30\%) e Trabalhos não baseados em PGs.

Foram consideradas as seguintes produções: artigos em periódicos, capítulo de livros, trabalhos completos em anais e resumos em congressos.

\section{RESULTADOS}

Dentre os 119 trabalhos realizados, apenas 4 PGs não foram incluídos nesta pesquisa, devida a dificuldade de contato com o orientador ou indisponibilidade do CV Lates atualizado. A lista dos PG, com seus autores e orientadores encontra-se anexo (ANEXO 1). Ao todo, os PGs foram orientados por 40 docentes, sendo que a exceção de um caso, todos eram professores do CTTMar. Os alunos foram orientados por professores do Curso de Oceanografia em $92 \%$ dos casos (Tab. 1). Embora não pertencentes ao curso, todos os orientadores externos tinham, em menor ou maio grau, relação com a Oceanografia. Considerando o quadro atual de docentes do curso, a média de orientação foi de 2,5 trabalhos por orientador. No entanto, esta distribuição dentre o total de docentes do curso não foi homogênea, variando de nenhuma até o máximo de 10 alunos orientados por um único professor.

Os PGs foram agrupados em seis grandes áreas do Curso de Oceanografia: Oceanografia Biológica, Oceanografia Geológica, Oceanografia Química, Oceanografia Física, Planejamento da Paisagem e Sensoriamento Remoto e Educação Ambiental (Fig. 1, ANEXO 1). A definição a qual grupo o PG pertencia foi baseada no tema e, em casos multidisciplinares, prevaleceu a origem do orientador do trabalho. Observa-se que o maior número de orientações ocorreu no âmbito da Oceanografia Biológica, com 70 casos, o que equivale a $59 \%$ dos trabalhos realizados. A Educação ambiental foi a área com menor número de trabalhos.

Em geral, os temas dos trabalhos foram bastante diversos. Dentro da Oc. Biológica, enfocaram desde a biologia de vegetais ou animais, a efeitos de substâncias tóxicas no desenvolvimento de organismos. A composição bioquímica também foi foco de trabalhos nesta área. Optou-se por agrupar os trabalhos de Planejamento da Paisagem e Sensoriamento Remoto, uma vez que estas disciplinas estão intimamente ligadas. Em grande parte dos trabalhos, o sensoriamento remoto estava associado a elaboração de um produto para subsídio potencial do planejamento, como por exemplo, taxas de ocupação do solo ou elaboração de produção de Sistemas de Informações Geográficas. Os trabalhos da Oc. Geológica foram mais expressivos na área de morfodinâmica ou geologia do quaternário. Já os projetos de Oc. Química e Física estiveram, em sua maior parte, relacionados a processos costeiros de dinâmica, principalmente ondas e de transporte, seja de sedimentos ou nutrientes. A poluição por metais também foi abordada, assim como o processo de eutrofização. Os trabalhos de Educação 
Tabela 1 - Resultados Gerais do Levantamento.

\begin{tabular}{l|c|c}
\hline \hline & Total & Percentagem \\
\hline PGs do curso até $20021^{\circ}$ Semestre & 119 & \\
\hline PGs incluídos na pesquisa & 115 & 96,6 \\
\hline Total de orientadores & 40 & \\
\hline Total de orientações do Curso & 110 & 92,4 \\
\hline Total de orientações do CTTMar & 118 & 99,2 \\
\hline Total de orientações fora do CTTMar & 1 & 0,8 \\
\hline Total de professores do Curso** & 48 & \\
\hline \hline
\end{tabular}

** Durante a elaboração deste trabalho

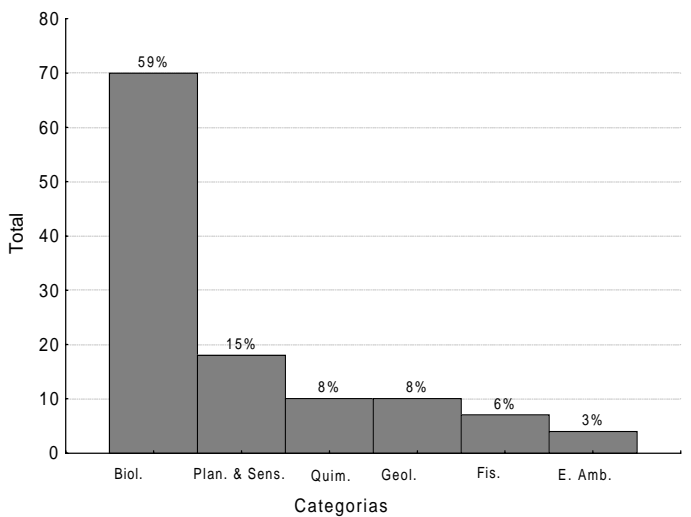

Figura 1 - Distribuição dos TCCs em 6 grandes áreas do Curso de Oceanografia (Biol.= Biológica; Plan \& Sens.= Planejamento da Paisagem e Sensoriamento Remoto; Quim.= Química; Geol.= Geologia; Fis. = Física e E. Amb.= Educação Ambiental.)

Ambiental envolveram diretamente temas relacionados à oceanografia, em suas diversas disciplinas.

A maior parte da produção científica gerada pelos PGs é relativa a resumos em congressos, com um total de 124 contribuições (Tabela 2), com uma média de 1,08 trabalhos por aluno. Somando-se todos os tipos de contribuição, tem-se que a média da contribuição de cada aluno é de 1,52 trabalhos. Cerca de um terço dos alunos não elaboraram nenhum tipo de publicação (Figura 2), conforme a metodologia utilizada e discutido abaixo. Por outro lado, um pequeno número de alunos publicou mais que cinco trabalhos derivados de seus PGs.

Considerando todos os graus de contribuição em trabalhos (total, na maior parte e em menor parte), verifica-se que em torno de $13 \%$ da produção total teve de alguma forma a contribuição dos PG (Tabela 3). Segundo os resultados, os PG são predominantemente apresentados na forma de resumos em congresso e capítulos em livro. Ressalta-se que, de acordo com a metodologia utilizada, a produção total do orientador foi computada e não somente no período de orientação.

\section{DISCUSSÃO}

Um primeiro ponto a ser levantado diz respeito à metodologia empregada para o levantamento da produção científica relacionada aos PGs. Um pressuposto desta pesquisa diz respeito à inclusão do orientador dos $\mathrm{PG}$ como co-autor dos trabalhos. Este pressuposto tem fundamento, uma vez que esta prática é comum entre os alunos de PG do curso de Oceanografia da UNIVALI (obs. pessoal). Outro pressuposto diz respeito à inclusão dos trabalhos envolvendo os PGs na lista de produção científica dos orientadores. Segundo o levantamento feito, os orientadores em geral incluem as colaborações com os alunos. Alguns no entanto, não consideram em seus CV as participações 
Tabela 2 - Produção científica relativas a 115 TCCs do Curso de Oceanografia, elaborados entre 1997 e 2002 (não incluídas participações em reuniões de iniciação científica).

\begin{tabular}{l|c|c|c|c|c}
\hline \hline & $\begin{array}{c}\text { Artigos } \\
\text { Periódicos }\end{array}$ & Capítulo de Llvros & $\begin{array}{c}\text { Trabalhos } \\
\text { Completos em } \\
\text { Anais }\end{array}$ & $\begin{array}{c}\text { Resumos em } \\
\text { Congressos }\end{array}$ & TOTAL \\
\hline Total & 17 & 6 & 29 & 124 & 176 \\
\hline Maximo/PG & 2 & 1 & 2 & 6 & 7 \\
\hline Média/PG & 0,15 & 0,05 & 0,24 & 1,08 & 1,52 \\
\hline \hline
\end{tabular}

relacionadas a iniciação científica, como participações em eventos direcionados a alunos de programas de iniciação científica, como por exemplo, o Programa Institucional de Bolsas de Iniciação Científica (Pibic) do CNPq. Este é o principal motivo da exclusão nesta pesquisa, da produção oriunda de eventos deste tipo. Um último pressuposto diz respeito à atualização da base de dados pelos orientadores. De acordo com a informação dos orientadores, a base de dados estava atualizada no momento da pesquisa.

O levantamento incluiu em torno de $97 \%$ dos PGs desenvolvidos, desde os primeiros trabalhos apresentados no Curso de Oceanografia, em julho 1997, até julho de 2002. A maior parte dos trabalhos foi orientada por professores do Curso de Oceanografia, seguindo do regulamento do PG (ProEn, 2003). A área da Oc. Biológica agrupou a maioria dos trabalhos, e sua dominância nos trabalhos de graduação reflete uma característica do Curso da UNIVALI. Em comparação com as outras, esta área é a mais representada no número de professores, pesquisadores e de disciplinas. A distribuição diferenciada entre as áreas também é observada em outros cursos, como o da Universidade Federal do Rio Grande, por exemplo (observação pessoal). O maior peso da biologia nos cursos de oceanografia é refletida também no número de trabalhos apresentados na XIV Semana Nacional de Oceanografia. A SNO é um evento de grande participação de estudantes, dos diferentes cursos de Oceanografia do país, onde parte da produção científica aqui relatada é publicada. Segundo a edição de 2001, foram 124 (61,0\%)

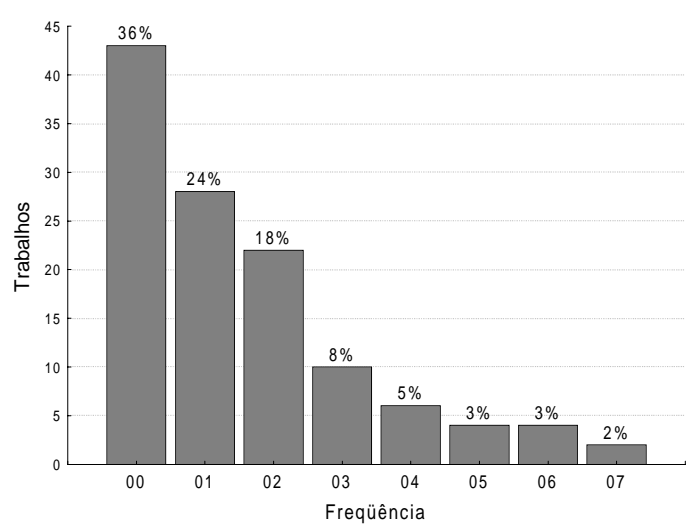

Figura 2 - Distribuição de freqüência da produção científica relativa a 115 PGs do Curso de Oceanografia, elaborados entre 1997 e 2002 (não incluídas participações em reuniões de iniciação científica).

trabalhos em Oc. Biológica, contra 27 (13,3\%) da Oc. Física, 24 (11,8\%) da Oc. Química, 17 $(8,4 \%)$ da Oc. Geológica e 11 (5,4) de Educação Ambiental (XIV SNO, 2001).

Os resultados do levantamento mostraram que aproximadamente dois terços dos alunos do Curso de Oceanografia produzem pelo menos uma publicação científica (latu sensu) fruto de seus PGs, sendo a média de 1,5 trabalhos por aluno. Ressalta-se que nesta pesquisa, apenas os trabalhos diretamente relacionados aos PGs foram computados, de outra forma, a produção científica dos alunos seria maior. Isto de deve ao fato de que os alunos do Curso de Oceanografia geralmente iniciam estágios junto aos laboratórios de pesquisa nos períodos iniciais (Coordenação Oceanografia, 2003). Esta característica faz com que no momento da elaboração de seus 
PROENÇA et al.: Projeto de graduação e a produção científica: Oceanografia CTTMar/UNIVALI.

Tabela 3 - Produção científica relativas a 115 PGs do Curso de Oceanografia, elaborados entre 1997 e 2002 em relação a produção dos orientadores (não incluídas participações em reuniões de iniciação científica).

\begin{tabular}{|c|c|c|c|c|c|}
\hline & $\begin{array}{c}\text { Artigos em } \\
\text { Periódicos } \\
(\%)\end{array}$ & $\begin{array}{l}\text { Capítulo de } \\
\text { Livros (\%) }\end{array}$ & $\begin{array}{c}\text { Trabalhos Completos } \\
\text { em Anais (\%) }\end{array}$ & $\begin{array}{c}\text { Resumos em } \\
\text { Congressos (\%) }\end{array}$ & Total (\%) \\
\hline $\begin{array}{l}\text { Totalmente baseados em } \\
\text { PGs }\end{array}$ & $10(3)$ & $6(8)$ & $18(6)$ & $78(8)$ & 112 \\
\hline $\begin{array}{l}\text { Parcialmente Baseados } \\
\text { em PGs }(>50 \%)\end{array}$ & $5(1)$ & $0(0)$ & $7(2)$ & $30(3)$ & 42 \\
\hline $\begin{array}{l}\text { Parcialmente Baseados } \\
\text { em PGs }(<50 \%)\end{array}$ & $2(1)$ & $0(0)$ & $4(2)$ & $16(1)$ & 22 \\
\hline TOTAL & 17 & 6 & 29 & 124 & 176 \\
\hline $\begin{array}{l}\text { TOTAL da produção dos } \\
\text { orientadores }\end{array}$ & $361(17)$ & $78(8)$ & 300 (10) & $1033(12)$ & $1772(10)$ \\
\hline
\end{tabular}

trabalhos de conclusão, muitos alunos já tenham sido iniciados na ciência, fazendo com que a média de contribuições seja relativamente alta.

Resumos em congressos foi a categoria mais expressiva em número de trabalhos. A participação em reuniões científicas é uma das principais vias de iniciação em ciência para os alunos. Sob orientação, os alunos aprendem a elaborar resumos, preparar painéis, apresentações orais e estarem prontos para discutir seus resultados. Este processo se diferencia da apresentação oral dos PGs, uma vez que aborda a questão científica do trabalho, e não o processo de aprendizagem em sí.

Embora se trate de produção científica, em termos de publicação, os resumos apresentados em reuniões científicas não são comparáveis a uma publicação em revista especializada, porém servem como uma primeira apresentação dos resultados de uma pesquisa. Geralmente, os resumos são aceitos sem restrições nas reuniões científicas, assegurando-se apenas a adequação do trabalho ao tema da reunião. Assim, justificase esta como a categoria com maior participação dos alunos.

Trabalhos completos em reuniões foi a segunda categoria com maior participação. Embora, muitas vezes os trabalhos completos não passem por revisores e sejam publicados conforme submetidos, sua elaboração requer a apresentação dos resultados e discussão, no formato exigido. A publicação em revistas especializadas demanda mais tempo. De acordo com a revista, os trabalhos antes de serem publicados, são enviados a revisores, especialistas no tema do trabalho, que a criticam em função das hipóteses, métodos empregados e discussão dos resultados. Este processo é geralmente lento e entre as idas e vindas do trabalho pode demorar mais que um ano até que seja publicado. Mesmo assim, PGs dos alunos de Oceanografia já contribuíram com mais do que 28 trabalhos publicados em revistas. Ressalta-se que, devido ao maior tempo para a publicação, é possível que este valor esteja subestimado, visto que alguns trabalhos possam estar ainda estar em processo de publicação.

Os PGs também contribuíram para a produção científica dos orientadores, uma vez que cerca de $10 \%$ da produção científica, aqui considerada, esteve a eles relacionada diretamente. Novamente, conforme a metodologia adotada, esta contribuição pode estar subestimada, uma vez que toda a produção do orientador foi computada, incluindo os trabalhos anteriores a período de orientação. Se considerarmos a produção de forma indireta ou difusa, esta contribuição pode ser ainda maior. Por exemplo, em muitos PGs 
metodologias foram adaptadas ou implementadas para serem posteriormente utilizadas em outros trabalhos, não necessariamente a eles relacionados.

Embora não esteja incluída nos objetivos da disciplina de Projeto de Graduação, os resultados aqui apresentados demonstram que a produção científica, e conseqüentemente, 0 conhecimento científico é incrementado com o desenvolvimento dos trabalhos de conclusão de curso.

\section{CONCLUSÕES}

A Oceanografia Biológica é a área dentro da qual o maior número de projetos de graduação foi desenvolvido, acompanhando a própria característica do Curso de Oceanografia. A disciplina de Projeto de Graduação é um instrumento de importante de iniciação científica para os alunos de Oceanografia da UNIVALI. Da mesma forma, a disciplina contribui de forma visível para a produção científica formal dos pesquisadores vinculados ao curso.

\section{REFERÊNCIAS BIBLIOGRÁFICAS}

Coordenação Oceanografia. 2003. Projeto pedagógico do Curso de Oceanografia, Universidade do Vale do Itajaí, Pró-Reitoria de Ensino,

http://lattes.cnpq.br/. Plataforma Lattes, Conselho Nacional de Desenvolvimento Científico e tecnológico, CNPq. Consultas entre Fevereiro e Março de 2003.

ProEn, 2002. Regulamentos do curso de oceanografia: regulamento de projeto de graduação e regulamento da atividade de embarque. Universidade do Vale do Itajaí, Pró-Reitoria de Ensino, $57 \mathrm{p}$.

http://www.cttmar.univali.br/ graduacao/. Projetos de Graduação Oceanografia. Consulta em fevereiro de 2003.
XIV SNO, 2001. Anais da XIV Semana Nacional de Oceanografia, 07 a 13 de outubro de 2001, Rio Grande, RS. Centro Acadêmico Livre de Oceanografia, CD rom. 
PROENÇA et al.: Projeto de graduação e a produção científica: Oceanografia CTTMar/UNIVALI.

Anexo 1 - Lista dos Projetos de Graduação do Curso de Oceanografia de apresentados entre agosto de 1997 e julho de 2002. Fonte: http://www.cttmar.univali.br/ graduacao.

\begin{tabular}{|c|c|}
\hline TCC No. 1 & Avaliação do Comportamento de Cobre e Chumbo Associado ao Material em Suapensão no Estuário do Rio Itajaí-açu - Brasil \\
\hline \multicolumn{2}{|c|}{$\begin{array}{l}\text { Autor(a): Túlio Alexandre da Costa } \\
\text { Orientador(a): MSc Kátia Naomi Kuroshima, Banca avaliadora: Dra. Eunice da Costa Machado, Dr. Paulo Baish } \\
\text { Data de Apresentação: } 14 / 08 / 1997\end{array}$} \\
\hline TCC No. 2 & Dinâmica de Nutrientes Inorgânicos no Estuário do Rio Itajaí-açu - SC \\
\hline \multicolumn{2}{|c|}{$\begin{array}{l}\text { Autor(a): Isabel Cristina Pellens Zacharjasiewicz } \\
\text { Orientador(a): MSc Valéria Regina Bellotto; Banca avaliadora: Dra. Eunice da Costa Machado, MSc. Jurandir Pereira Filho } \\
\text { Data de Apresentação: } 14 / 08 / 1997\end{array}$} \\
\hline TCC No. 3 & Dinâmica de Nutrientes Inorgânicos na Enseada de Camboriú - SC \\
\hline \multicolumn{2}{|c|}{$\begin{array}{l}\text { Autor(a): Fabiano Morelli } \\
\text { Orientador(a): MSc Valéria Regina Bellotto; Banca avaliadora: Dra. Eunice da Costa Machado, MSc. Jurandir Pereira Filho } \\
\text { Data de Apresentação: } 14 / 08 / 1997\end{array}$} \\
\hline TCC No. 4 & Oscilações do Nível Médio do Mar e as Cheias do Rio Itajaí-açu. \\
\hline \multicolumn{2}{|c|}{$\begin{array}{l}\text { Autor(a): Pablo Medeiros Jabor } \\
\text { Orientador(a): MSc João Luiz Baptista de Carvalho; Banca avaliadora: MSc Renato Parkinson, MSc Carlos Augusto F. Schettini } \\
\text { Data de Apresentação: 15/08/1997 }\end{array}$} \\
\hline TCC No. 5 & $\begin{array}{c}\text { Monitoramento do Índice de condição de Mexilhões Perna perna no Parque de Mitilicultura na Enseada de Armação do } \\
\text { Itapocoroy - Penha, SC }\end{array}$ \\
\hline \multicolumn{2}{|c|}{$\begin{array}{l}\text { Autor(a): Eduardo Wojciechowiski Junior } \\
\text { Orientador(a): MSc Adriano Weidner Cacciatori Marenzi; Banca avaliadora: MSc Paulo R. Pezzutto, MSc Gilberto C. Manzoni } \\
\text { Data de Apresentação: } 15 / 12 / 1997\end{array}$} \\
\hline TCC No. 6 & Dinâmica da Matéria Orgânica do Estuário do Rio Itajaí-açu, SC. \\
\hline \multicolumn{2}{|c|}{$\begin{array}{l}\text { Autor(a): Patrícia Fóes Scherer } \\
\text { Orientador(a): MSc Kátia Naomi Kuroshima; Banca avaliadora: MSc. Jurandir Pereira Filho, MSc. Leonardo R. Rörig } \\
\text { Data de Apresentação: } 15 / 12 / 1997\end{array}$} \\
\hline TCC No. 7 & $\begin{array}{c}\text { Análise dos Nichos Ecológicos na Pesca com Redes de Emalhe na Comunidade Pesqueira Artesanal da Praia da Canoa (Barra } \\
\text { Velha - SC) }\end{array}$ \\
\hline \multicolumn{2}{|c|}{$\begin{array}{l}\text { Autor(a): Rodrigo Pereira Medeiros } \\
\text { Orientador(a): Dr Marcus Polette; Banca avaliadora: MSc Dagmar von Linsingen, Dr. José Angel A. Perez } \\
\text { Data de Apresentação: } 15 / 12 / 1997\end{array}$} \\
\hline TCC No. 8 & Sistemática e Biologia dos Myxini do Litoral Brasileiro \\
\hline \multicolumn{2}{|c|}{$\begin{array}{l}\text { Autor(a): Michael Maia } \\
\text { Orientador(a): Dr. José } \\
\text { Data de Apresentação: }\end{array}$} \\
\hline TCC No. 9 & $\begin{array}{c}\text { Crescimento da Garopa Epinephelus marginatus (=Guaza) (Osteichtyes - Serranidae) em Tamque-Rede, no Município de } \\
\text { Penha, SC. }\end{array}$ \\
\hline \multicolumn{2}{|c|}{$\begin{array}{l}\text { Autor(a): Norberto Ghizoni } \\
\text { Orientador(a): MSc Maurício Hostim Silva; Banca avaliadora: Eng. Celso F. Lin, MSc. Gilberto C. Manzoni } \\
\text { Data de Apresentação: } 16 / 12 / 1997\end{array}$} \\
\hline TCC No. 10 & $\begin{array}{c}\text { A Criação da Reserva Marinha do Arvoredo (SC) e suas Implicações sobre as Atividades Pesqueiras Artesanais das Comunidades } \\
\text { de Zimbros e Canto Grande }\end{array}$ \\
\hline \multicolumn{2}{|c|}{$\begin{array}{l}\text { Autor(a): Gabriel Botelho Marchioro } \\
\text { Orientador(a): Dr Marcus Polette; Banca avaliadora: Oc. Roberto Wahrlich, Oc. José Matarezi } \\
\text { Data de Apresentação: 16/07/1998 }\end{array}$} \\
\hline TCC No. 11 & $\begin{array}{c}\text { Estudo Quali-quantitativo da Fauna Acompanhante do Camarão Sete Barbas Xiphopenaues kroyeri (Heller, 1862) na Enseada } \\
\text { de Armação do Itapocoroy, Penha, SC. }\end{array}$ \\
\hline \multicolumn{2}{|c|}{$\begin{array}{l}\text { Autor(a): Rodrigo Schveitzer } \\
\text { Orientador(a): Dr Joaquim Olinto Branco; Banca avaliadora: MSc Luiz Eduardo Bonilha, Oc. Roberto Wahrlich } \\
\text { Data de Apresentação: } 16 / 07 / 1998\end{array}$} \\
\hline TCC No. 12 & $\begin{array}{c}\text { Implantação e Manutenção de Cultivos de Dinoflagelados Marinhos do Gênero Prorocentrum e Determinação de Taxas de } \\
\text { Crescimento a Diferentes Salinidades. }\end{array}$ \\
\hline \multicolumn{2}{|c|}{$\begin{array}{l}\text { Autor(a): Sílvio César Pereira Guimarães } \\
\text { Orientador(a): MSc Leonardo Rubi Rörig; Banca avaliadora: MSc. Simone Rabelo da Cunha, MSc. Charrid Resgalla Jr } \\
\text { Data de Apresentação: } 17 / 07 / 1998\end{array}$} \\
\hline TCC No. 13 & $\begin{array}{c}\text { Monitoramento Bacteriológico da Água e dos Moluscos Cultivados na Enseada de Armação do Itapocoroy - Penha - Santa } \\
\text { Catarina - Brasil, Utilizando Coliformes Fecais como Bioindicadores de Poluição. }\end{array}$ \\
\hline \multicolumn{2}{|c|}{$\begin{array}{l}\text { Autor(a): Jean Franco Schmitt } \\
\text { Orientador(a): MSc Gilberto Caetano Manzoni; Banca avaliadora: MSc. Alexandre Brandelli, } \\
\text { Data de Apresentação: } 17 / 07 / 1998\end{array}$} \\
\hline TCC No. 14 & Análise de Ecologia da Paisagem em Ilhas do Litoral do Estado de Santa Catarina. \\
\hline
\end{tabular}


Anexo 1 - Continuação.

\begin{tabular}{|c|c|}
\hline \multirow{2}{*}{\multicolumn{2}{|c|}{$\begin{array}{l}\text { TCC No. } 15 \\
\text { Autor(a): Sílvio Souza Júnior } \\
\text { Orientador(a): Dr Marcus Polette; Banca avalo do Modelo de Desenvolvimento de Balneários no Município de Balneário Camboriú (S } \\
\text { Data de Apresentação: 08/12/1998 }\end{array}$}} \\
\hline & \\
\hline TCC No. 16 & Perfil da Frota Pesqueira e do Parque Industrial de Itajaí e Navegantes \\
\hline \multicolumn{2}{|c|}{$\begin{array}{l}\text { Autor(a): Fábio Alexandre Travassos } \\
\text { Orientador(a): MSc Roberto Wahrlich; Banca avaliadora: Oc. Agostinho Peruzzo, Oc. Marco Aurélio Bailon } \\
\text { Data de Apresentação: }\end{array}$} \\
\hline TCC No. 17 & Determinação de Metais Pesados em Mexilhões Cultivados na Enseada de Zimbros \\
\hline \multicolumn{2}{|c|}{$\begin{array}{l}\text { Autor(a): Fernanda Freyeslebem Thomazelli } \\
\text { Orientador(a): MSc Kátia Naomi Kuroshima; Banca avaliadora: MSc. Valéria Regina Bellotto, MSc. Gilberto Caetano Manzoni } \\
\text { Data de Apresentação: 09/12/1998 }\end{array}$} \\
\hline TCC No. 18 & $\begin{array}{c}\begin{array}{c}\text { Relação entre o Comprimento das Rizinas e a Taxa de Crescimento da Macroalga Gelidium sesquipedale (Clem.) Bprn. et Thur } \\
\text { em Albufeira - Portugal. }\end{array} \\
\end{array}$ \\
\hline \multicolumn{2}{|c|}{$\begin{array}{l}\text { Autor(a): André Luiz Richter Nascimento } \\
\text { Orientador(a): MSc Mônica Maria Pereira Tognella De Rosa; Banca avaliadora: MSc. Simone Rabelo da Cunha, MSc. Gilberto Caetano Manzoni } \\
\text { Data de Apresentação: 09/12/1998 }\end{array}$} \\
\hline TCC No. 19 & $\begin{array}{c}\text { Exploração de Dados de Comprimento em Estudos de Estoques Pesqueiros: o Caso do Bonito Listrado (Katsuvomus pelamis) } \\
\text { Capturado na Costa do Brasil }\end{array}$ \\
\hline \multicolumn{2}{|c|}{$\begin{array}{l}\text { Autor(a): Rodrigo de Oliveira Campos } \\
\text { Orientador(a): MSc Humber Agrelli de Andrade; Banca avaliadora: Dr. José Angel Alvarez Perez, Eng. Pesca Celso Lin } \\
\text { Data de Apresentação: 09/12/1998 }\end{array}$} \\
\hline TCC No. 20 & Aspectos Morfodinâmicos das Praias do Litoral Centro-Norte Catarinense \\
\hline \multicolumn{2}{|c|}{$\begin{array}{l}\text { Autor(a): João Thadeu de Menezes } \\
\text { Orientador(a): MSc Antonio Henrique da Fontoura Klein; Banca avaliadora: Dr. Rodolfo Ângulo, MSc. João Luiz Baptista de Carvalho } \\
\text { Data de Apresentação: 13/04/1999 }\end{array}$} \\
\hline TCC No. 21 & Estudo da Estabilidade da Barra do Camacho, Santa Catarina, Brasil \\
\hline \multicolumn{2}{|c|}{$\begin{array}{l}\text { Autor(a): Daniella Borderes de Oliveira } \\
\text { Orientador(a): MSc João Luiz Baptista de Carvalho; Banca avaliadora: MSc. Milton Sant'ana, MSc. Carlos Augusto França Schettini } \\
\text { Data de Apresentação: 17/06/1999 }\end{array}$} \\
\hline TCC No. 22 & Composição Bioquímica da Vieira Pecten ziczac em Distintas Fases Reprodutivas \\
\hline \multicolumn{2}{|c|}{$\begin{array}{l}\text { Autor(a): Karine Marie Arasaki } \\
\text { Orientador(a): MSc Marcos Luiz Pessatti; Banca avaliadora: MSc. Paulo Ricardo Pezzuto, MSc. Gilberto Caetano Manzoni } \\
\text { Data de Apresentação: } 17 / 06 / 1999\end{array}$} \\
\hline TCC No. 23 & $\begin{array}{c}\text { Implantação de Cultivos Unialgais das Diatomáceas Asterionellopsis Glacialis e Anaulus australis e suas Carac } \\
\text { Crescimento à Diferentes Salinidades }\end{array}$ \\
\hline \multicolumn{2}{|c|}{$\begin{array}{l}\text { Autor(a): Carolina Ximenes de Macedo } \\
\text { Orientador(a): MSc Leonardo Rubi Rörig; Banca avaliadora: Dr. Luis Antonio de Olivieira Proença, MSc. Gilberto Caetano Manzoni } \\
\text { Data de Apresentação: } 18 / 06 / 1999\end{array}$} \\
\hline TCC No. 24 & $\begin{array}{c}\text { Determinação dos Fatores Ambientais Atuantes na Variação do Índice de Condição do Mexilhão Perna perna em Cultivo da } \\
\text { Enseada de Armaça do Itapocoroy, Penha, SC }\end{array}$ \\
\hline \multicolumn{2}{|c|}{$\begin{array}{l}\text { Autor(a): Rodrigo Otávio de Macedo Gomes } \\
\text { Orientador(a): MSc Adriano Weidner Cacciatori Marenzi; Banca avaliadora: MSc. Luiz Eduardo Carvalho Bonilha, MSc. Gilberto Caetano Manzoni } \\
\text { Data de Apresentação: } 18 / 06 / 1999\end{array}$} \\
\hline TCC No. 25 & $\begin{array}{c}\text { Análise Cartográfica das Mudanças da Paisagem Costeira no Município de Bombinhas (SC) Considerando a Evolução dos } \\
\text { Planos Diretores entre o Período } 1992 \text { e } 1996\end{array}$ \\
\hline \multicolumn{2}{|c|}{$\begin{array}{l}\text { Autor(a): Mônica Cruz Guerreiro } \\
\text { Orientador(a): Dr Marcus Polette; Banca avaliadora: MSc. Josildete de Oliveira, MSc. Paulo Pires } \\
\text { Data de Apresentação: } 18 / 06 / 1999\end{array}$} \\
\hline TCC No. 26 & Estudos Preliminares Envolvendo Testes de Toxicidade Embrio-Larvais com o Mexilhão Perna perno \\
\hline \multicolumn{2}{|c|}{$\begin{array}{l}\text { Autor(a): Ricardo Wagner Reis Filho; } \\
\text { Orientador(a): MSc Charrid Resgalla Júnior; Banca avaliadora: MSc. Leonardo Rubi Rörig, MSc. Gilberto Caetano Manzoni } \\
\text { Data de Apresentação: 18/11/1999 }\end{array}$} \\
\hline TCC No. 27 & Avaliação da Dinâmica Lateral e dos Mecanismos de Transporte no Estuário do Rio Camboriú \\
\hline \multicolumn{2}{|c|}{$\begin{array}{l}\text { Autor(a): Leandro Franklin da Silva } \\
\text { Orientador(a): MSc Carlos Augusto França Schettini; Banca avaliadora: Dr. Jarbas Bonetti, MSc. Antônio Henrique da Fontoura Klein } \\
\text { Data de Apresentação: 18/11/1999 }\end{array}$} \\
\hline TCC No. 28 & Estudo da Influência da Descarga Fluvial na Progressão da Onda de Maré no Estuário do Rio Itajaí-açu -SC \\
\hline \multicolumn{2}{|c|}{$\begin{array}{l}\text { Autor(a): Stênio dos Santos Stein } \\
\text { Orientador(a): MSc Carlos Augusto França Schettini; Banca avaliadora: MSc. Eliane Cristina Truccolo, MSc. Franklin Misael Pacheco Tena } \\
\text { Data de Apresentação: 18/11/1999 }\end{array}$} \\
\hline TCC No. 29 & Aspectos Reprodutivos de Stellifer rastrifer (Jordan, 1889) (Perciformes - Sciaenidae) na Foz do Rio Itajai-açu, SC, Brasil \\
\hline
\end{tabular}


PROENÇA et al.: Projeto de graduação e a produção científica: Oceanografia CTTMar/UNIVALI.

Anexo 1 - Continuação.

\begin{tabular}{|c|c|}
\hline TCC No. 30 & Guia em CD-Rom para Estudos das Aves Marinhas do Estado de Santa Catarina \\
\hline \multicolumn{2}{|c|}{$\begin{array}{l}\text { Autor(a): Antônio Henrique Roman } \\
\text { Orientador(a): Dr Joaquim Olinto Branco; Banca avaliadora: Dr. Oldemar Carvalho Junior, MSc. Adriano Weidner Cacciatori Marenzi } \\
\text { Data de Apresentação: } 18 / 11 / 1999\end{array}$} \\
\hline TCC No. 31 & Ocorrência de Dinoflagelados do Gênero Dinophysis (Enrenberg, 1839) na Enseada de Cabeçudas (verão e outono de 1999) \\
\hline \multicolumn{2}{|c|}{$\begin{array}{l}\text { Autor(a): Fabrício Schmitt } \\
\text { Orientador(a): Dr Luís Antônio de Oliveira Proença; Banca avaliadora: MSc. Leonardo Rubi Rörig, MSc. Katia Naomi Kuroshima } \\
\text { Data de Apresentação: 19/11/1999 }\end{array}$} \\
\hline TCC No. 32 & $\begin{array}{l}\text { Alimentação Natural de Callinectes sapidus Rathbun, } 1896 \text { e Callinectes danae Smith, no Estuário do Saco da Fazenda, Itajaí, } \\
\text { SC }\end{array}$ \\
\hline \multicolumn{2}{|c|}{$\begin{array}{l}\text { Autor(a): Willian Guimarães Vale } \\
\text { Orientador(a): Dr Joaquim Olinto Branco; Banca avaliadora: MSc. Silvia Helena Bulizane Lucato, MSc. Luiz Eduardo Carvalho Bonilha } \\
\text { Data de Apresentação: 19/11/1999 }\end{array}$} \\
\hline TCC No. 33 & $\begin{array}{c}\text { Determinação do Efeito do Esgoto Bruto e Efluente da Estação de Tratamento de Esgotos de Balneário Camboriú sobre o } \\
\text { Crescimento de Skeletonema costatum (Baccilaiophyceae) }\end{array}$ \\
\hline \multicolumn{2}{|c|}{$\begin{array}{l}\text { Autor(a): Ângela Zaccaron da Silva } \\
\text { Orientador(a): MSc Leonardo Rubi Rörig; Banca avaliadora: MSc Charrid Resgala Jr, MSc Kátia Naomi Kuroshima } \\
\text { Data de Apresentação: 19/11/1999 }\end{array}$} \\
\hline TCC No. 34 & $\begin{array}{c}\text { Utilização do Pacu Piaractus mesopotamicus (Holmberg 1887), em Sistema de Cultivo Semi Intensivo. Uma Alte } \\
\text { Aproveitamento de Espécie Alóctone, para as Condiçôes de Clima da Região Sul do Brasil }\end{array}$ \\
\hline \multicolumn{2}{|c|}{$\begin{array}{l}\text { Autor(a): Hugo Ricardo Lamas Diogo } \\
\text { Orientador(a): Dr Hilton Amaral Júnior; Banca avaliadora: Dr. Joaquim Olinto Branco, MSc. Gilberto Caetano Manzoni } \\
\text { Data de Apresentação: 19/11/1999 }\end{array}$} \\
\hline TCC No. 35 & Avaliação das Características Químicas em uma Área de Cultivo de Moluscos: Enseada de Armação do Itapocoroy, Penha-SC \\
\hline \multicolumn{2}{|c|}{$\begin{array}{l}\text { Autor(a): Gabriela Garbinatto Chevarria } \\
\text { Orientador(a): MSc Kátia Naomi Kuroshima; Banca avaliadora: MSc Gilberto Caetano Manzoni, Dr. Luis Antonio de Oliveira Proença } \\
\text { Data de Apresentação: 19/11/1999 }\end{array}$} \\
\hline TCC No. 36 & Geologia da Planície Costeira do Sistema Praial Brava-Amores, Santa Catarina, Brasil \\
\hline \multicolumn{2}{|c|}{$\begin{array}{l}\text { Autor(a): Armand Hanna Amin Júnior } \\
\text { Orientador(a): MSc Norberto Horn Filho; Banca avaliadora: Dr. Francisco Caruso Gomes Junior, , MSc Fernando Luiz Diehl } \\
\text { Data de Apresentação: }\end{array}$} \\
\hline TCC No. 37 & $\begin{array}{c}\text { Subsídios ao Ordenamento do Espaço Marinho da Enseada da Armação do Itapocoroy (Penha - SC), a Fim de Mitigar os } \\
\text { Ccnflitos Gerados pela Maricultura }\end{array}$ \\
\hline \multicolumn{2}{|c|}{$\begin{array}{l}\text { Autor(a): Leocádio Neves e Silva } \\
\text { Orientador(a): Dr Marcus Polette; Banca avaliadora: MSc. Gilberto Caetano Manzoni, Dr. Luís Antônio de Oliveira Proença } \\
\text { Data de Apresentação: 16/06/2000 }\end{array}$} \\
\hline TCC No. 38 & Proposta de Produção de um CD-ROM para o Comitê do Rio Camboriú, SC Planejamento da Pa \\
\hline \multicolumn{2}{|c|}{$\begin{array}{l}\text { Autor(a): Cláudio Rogério Guerra } \\
\text { Orientador(a): Dr Marcus Polette; Banca avaliadora: MSc. Leonardo Rubi Rörig, Oc. Patricia Wegner } \\
\text { Data de Apresentação: } 16 / 06 / 2000\end{array}$} \\
\hline TCC No. 39 & $\begin{array}{c}\text { Usos de Testes de Toxicidade com Larvas de Arbacea lixula e Naupliu de Artemia salina na Avaliação da Qualidade do } \\
\text { Sedimento das Desembocaduras dos Rios Itajai-açu, Itapocu e Tijucas }\end{array}$ \\
\hline \multicolumn{2}{|c|}{$\begin{array}{l}\text { Autor(a): Kalinka dos Santos Laitano } \\
\text { Orientador(a): MSc Charrid Resgalla Júnior; Banca avaliadora: MSc. Valeria Regina Bellotto, MSc. Leonardo Rubi Rörig } \\
\text { Data de Apresentação: } 16 / 06 / 2000\end{array}$} \\
\hline TCC No. 40 & Avaliação da Captura e Esforço Empregado pela Frota de Parelhas na Pesca Industrial de Itajaí - SC \\
\hline \multicolumn{2}{|c|}{$\begin{array}{l}\text { Autor(a): Anderson Ayres Pelanda } \\
\text { Orientador(a): Dr Paulo Ricardo Schwingel; Banca avaliadora: Dr. José Angel Alvarez Perez, MSc. Humber Agrelli } \\
\text { Data de Apresentação: 16/06/2000 }\end{array}$} \\
\hline TCC No. 41 & Alimentação Natural de Paralonchurus brasiliensis Steindachner, 1875, na Armação do Itapocoroy, Penha, SC. \\
\hline \multicolumn{2}{|c|}{$\begin{array}{l}\text { Autor(a): Flávio Xavier Souto } \\
\text { Orientador(a): Dr Joaquim Olinto Branco; Banca avaliadora: MSc Adriano Weidner Cacciatori Marenzi, MSc Maria José Lunardon-Branco } \\
\text { Data de Apresentação: 19/06/2000 }\end{array}$} \\
\hline TCC No. 42 & Avaliação da Qualidade da Água Utilizada em Diferentes Tanques de Piscicultura Localizados na EPAGRI de Cambo \\
\hline \multicolumn{2}{|c|}{$\begin{array}{l}\text { Autor(a): Uirá Cavalcante Oliveira } \\
\text { Orientador(a): MSc Kátia Naomi Kuroshima; Banca avaliadora: Dr. Luís Antonio de Oliveira Proença, MSc. Jurandir Pereira Filho } \\
\text { Data de Apresentação: } 19 / 06 / 2000\end{array}$} \\
\hline TCC No. 43 & $\begin{array}{c}\text { Estudo da Abundância Relativa de Juvenis de Garoupas (Epinephelus marginatus, Lowe 1834) (Perciformes: Serranidae, } \\
\text { Epinephelinae) no Costão da Sepultura - Bombinhas - SC - BR }\end{array}$ \\
\hline \multicolumn{2}{|c|}{$\begin{array}{l}\text { Autor(a): Leonardo Francisco Machado } \\
\text { Orientador(a): MSc Maurício Hostim Silva; Banca avaliadora: Dr José Angel Alvarez Perez, MSc Luiz Fernando Duboc da Silva } \\
\text { Data de Apresentação: } 19 / 06 / 2000\end{array}$} \\
\hline TCC No. 44 & Taxonomia e variação de larvas de cirripedia na enseada de Itapocoroy, Armação, Santa Catarina \\
\hline
\end{tabular}


Anexo 1 - Continuação.

\begin{tabular}{|c|c|}
\hline TCC No. 45 & $\begin{array}{c}\text { O Uso de Sistemas Informações Geográficas (SIG) na Caracterização Ambiental da Porção Sudeste do Município de Balneário } \\
\text { Camboriú - SC }\end{array}$ \\
\hline \multicolumn{2}{|c|}{$\begin{array}{l}\text { Autor(a): Luiz Fernando Raizer Moro } \\
\text { Orientador(a): MSc Rosana Maria Rodrigues; Banca avaliadora: Dr. Oldemar Carvalho Jr., MSc. Mônica Maria Pereira Tognella De Rosa } \\
\text { Data de Apresentação: 20/06/2000 }\end{array}$} \\
\hline TCC No. 46 & Efeito de Diferentes Salinidades no Crescimento e Produção de Toxinas pelo Dinoflagelado Gymnodinium catenatum \\
\hline \multicolumn{2}{|c|}{$\begin{array}{l}\text { Autor(a): Nagib Paulo de Souza } \\
\text { Orientador(a): Dr Luís Antônio de Oliveira Proença; Banca avaliadora: MSc Leonardo Rubi Rörig, MSc Adriano Weidner Cacciatori Marenzi } \\
\text { Data de Apresentação: 20/06/2000 }\end{array}$} \\
\hline TCC No. 47 & Monitoramento do Índice Gônado Somático do Ouriço-do-mar, Echinometra lucunter \\
\hline \multicolumn{2}{|c|}{$\begin{array}{l}\text { Autor(a): Natascha Tye Tamaki } \\
\text { Orientador(a): MSc Adriano Weidner Cacciatori Marenzi; Banca avaliadora: Dr. Joaquim Olinto branco, MSc. Gilberto caetano Manzoni } \\
\text { Data de Apresentação: 20/06/2000 }\end{array}$} \\
\hline TCC No. 48 & Caracterização Sedimentológica de Areias para Engordamento da Praia de Gravatá, Município de Navegantes \\
\hline \multicolumn{2}{|c|}{$\begin{array}{l}\text { Autor(a): Lourival Anastácio Alves Júnior } \\
\text { Orientador(a): MSc Maria Inês Freitas dos Santos; Banca avaliadora: MSc. José Gustavo Natorf de Abreu, Dr. Francisco Caruso Jr } \\
\text { Data de Apresentação: 20/06/2000 }\end{array}$} \\
\hline TCC No. 49 & Avaliação do Potencial de Crescimento In Vitro da Alga Vermelha Gracilaria caudata \\
\hline \multicolumn{2}{|c|}{$\begin{array}{l}\text { Autor(a): Débora Ortiz Lugli } \\
\text { Orientador(a): MSc Mônica Maria Pereira Tognella De Rosa; Banca avaliadora: MSc Gilberto Caetano Manzoni, Dra Zenilda Bouzon } \\
\text { Data de Apresentação: 21/06/2000 }\end{array}$} \\
\hline TCC No. 50 & Marina Veleiros da Ilha: uma Análise Descritiva Baseada em Parâmetros Físicos, Químicos e Biológi \\
\hline \multicolumn{2}{|c|}{$\begin{array}{l}\text { Autor(a): Sebastião Weiber Cavalari Júnior } \\
\text { Orientador(a): Dr Oldemar de Oliveira Carvalho Júnior; Banca avaliadora: Dr. Francisco Caruzo Jr., Claudio Amaral } \\
\text { Data de Apresentação: } 21 / 06 / 2000\end{array}$} \\
\hline TCC No. 51 & $\begin{array}{c}\text { Caracterização Estrutural do manguezal do Rio Tavares e Levantamento Sócio-econômico e Cultural da Comunidade Associada } \\
\text { a Reserva Estrativista Marinha do Pirajubaé (RESEX do Pirajubaé) (Baía Sul-Florianópolis-Santa Catarina) }\end{array}$ \\
\hline \multicolumn{2}{|c|}{$\begin{array}{l}\text { Autor(a): Samanta Carnavarolo Vizinho } \\
\text { Orientador(a): MSc Mônica Maria Pereira Tognella De Rosa; Banca avaliadora: MSc Luiz eduardo Carvalho Bonilha, Biol. Claudia Regina Santos } \\
\text { Data de Apresentação: } 21 / 06 / 2000\end{array}$} \\
\hline TCC No. 52 & $\begin{array}{c}\text { Crescimento e Sobrevivência da Vieira Nodipecten nodosus (Linnaeus, 1758) (Mollusca: Bivalvia) em Cultivo Experimental na } \\
\text { Enseada da Armação do Itapocoroy (Penha - SC - Brasil) }\end{array}$ \\
\hline \multicolumn{2}{|c|}{$\begin{array}{l}\text { Autor(a): Gisele Cristina Baldissera } \\
\text { Orientador(a): MSc Gilberto Caetano Manzoni; Banca avaliadora: MSc. Adriano Weidner Cacciatori Marenzi, Dr. José Angel Alvarez Perez } \\
\text { Data de Apresentação: } 21 / 06 / 2000\end{array}$} \\
\hline TCC No. 53 & $\begin{array}{c}\text { Estimativa do Potencial de Aproveitamento da Fauna Acompanhante (Amphipoda - Polichaeta) dos Cultivos de Mexill } \\
\text { Municipio de Penha-SC }\end{array}$ \\
\hline \multicolumn{2}{|c|}{$\begin{array}{l}\text { Autor(a): Anderson Ricardo Voos } \\
\text { Orientador(a): MSc Charrid Resgalla Júnior; Banca avaliadora: MSc. Paulo Ricardo Pezzuto, MSc. Gilberto Caetano Manzoni } \\
\text { Data de Apresentação: } 21 / 06 / 2000\end{array}$} \\
\hline TCC No. 54 & Análise das Metodologias para o Mapeamento da Ambientes de Sedimentação Costeira \\
\hline \multicolumn{2}{|c|}{$\begin{array}{l}\text { Autor(a): Henrique Frasson de Souza Mário } \\
\text { Orientador(a): Dr Francisco Caruso Gomes Júnior; Banca avaliadora: MSc Sérgio Freitas Borges, MSc Luis Fernando Pedroso Sales } \\
\text { Data de Apresentação: } 21 / 11 / 2000\end{array}$} \\
\hline TCC No. 55 & $\begin{array}{c}\text { Análise Comparativa das Embarcações de Espinheleiros Nacionais e Arrendados na Região Sul e Sudeste do Brasil: Subsídios } \\
\text { para o Gerenciamento da Pesca. }\end{array}$ \\
\hline \multicolumn{2}{|c|}{$\begin{array}{l}\text { Autor(a): Marcelo Barbosa Sampaio } \\
\text { Orientador(a): MSc Roberto Wahrlich; Banca avaliadora: Dr. Paulo Ricardo Schuwingel, Dr. José Angel Alvares Perez } \\
\text { Data de Apresentação: } 21 / 11 / 2000\end{array}$} \\
\hline TCC No. 56 & $\begin{array}{c}\text { Estrutura Populacional de Stellifer spp (Pecrciformes, Sciaenidae), na Pesca Artesanal do Camarão Sete-B } \\
\text { Itapocoroy, Penha, SC }\end{array}$ \\
\hline \multicolumn{2}{|c|}{$\begin{array}{l}\text { Autor(a): Laura Ribas de Almeida } \\
\text { Orientador(a): Dr Joaquim Olinto Branco; Banca avaliadora: Dr. José Angel Alvarez Perez, MSc Maurício Hostim Silva } \\
\text { Data de Apresentação: } 21 / 11 / 2000\end{array}$} \\
\hline TCC No. 57 & $\begin{array}{c}\text { Distribuição Espacial e Abundância do Gastrópode Thais (Stramonita) haemastoma (Linaeus, 1767) no Mesolitoral de Co } \\
\text { Rochosos do Litoral Centro Norte Catarinense (Balneário Camboriú - Penha) }\end{array}$ \\
\hline \multicolumn{2}{|c|}{$\begin{array}{l}\text { Autor(a): Luciano Antônio Lacava } \\
\text { Orientador(a): MSc Gilberto Caetano Manzoni; Banca avaliadora: Adriano Weidner Cacciatori Marenzi, Dr. Joaquim Olinto Branco } \\
\text { Data de Apresentação: } 21 / 11 / 2000\end{array}$} \\
\hline TCC No. 58 & $\begin{array}{c}\text { Análise dos Desembarques do Pitú ou Lagostim Metanephrops rubellus (Moreira, 1903) (Decápoda: Astacidea:Nephropidae) } \\
\text { efetuados pela frota camaroeira em Santos (SP) e Itajaí (SC) }\end{array}$ \\
\hline \multicolumn{2}{|c|}{$\begin{array}{l}\text { Autor(a): Thais Mehl Ribas } \\
\text { Orientador(a): MSc Paulo Ricardo Pezzuto; Banca avaliadora: Dr José Angel Alvarez Perez, MSc Roberto Wahrlich } \\
\text { Data de Apresentação: 21/11/2000 }\end{array}$} \\
\hline TCC No. 59 & Distribuição e Especiação de Metais-Traço nos Sedimentos da Plataforma Continental de Santa Catarina \\
\hline
\end{tabular}


PROENÇA et al.: Projeto de graduação e a produção científica: Oceanografia CTTMar/UNIVALI.

Anexo 1 - Continuação.

\begin{tabular}{|l|ll}
\hline TCC No. 60 & Papel das Ondas e Correntes na Remobilização de Sedimentos em Zonas de Cultivo de Moluscos Marinhos.
\end{tabular}

Autor(a): Carla de Abreu D’Aquino

Orientador(a): MSc Carlos Augusto França Schettini; Banca avaliadora: MSc Gilberto Caetano Manzoni, Dr. Elirio Toldo Jr

Data de Apresentação: 22/11/2000

TCC No. $61 \quad$ Tolerância a Salinidade da Tilápia Vermelha (Oreochromis sp), Tilápia Careca (Oreochromis sp) e o Híbrido destas Espécies

Autor(a): Luciano Strefling

Orientador(a): Dr Gosuke Sato; Banca avaliadora: MSc Marcelo Rodrigues Riobeiro, MSc. Gilberto Caetano Manzoni

Data de Apresentação: 22/11/2000

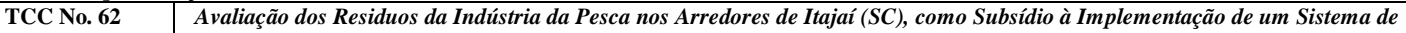

Autor(a): Fernanda Terra Stori Gerencial de Bolsa de Resíduos

Orientador(a): MSc Luiz Eduardo Carvalho Bonilha; Banca avaliadora: MSc. Roberto Wahrlich, Dr. Alexandre Lerípio

Data de Apresentação: 22/11/2000

TCC No. 63 Avaliação da Estrutura do Gladius de Loligo plei (Mollusca: Cephalopoda) Para Estudos de Idade e Crescimento.

Autor(a): João Antônio P. R. T. dos Santos

Orientador(a): Dr José Angel Alvarez Perez; Banca avaliadora: MSc Paulo Ricardo Pezzuto, MSc Humber Agrelli de Andrade

Data de Apresentação: 22/11/2000

TCC No. 64 Um Estudo do clima de Ondas para a Região de Cabo Frio

Autor(a): Janini Pereira

Orientador(a): MSc João Luiz Baptista de Carvalho; Banca avaliadora: MSc Rogério N. Candela, MSc Eliane Cristina Truccolo

Data de Apresentação: 23/11/2000

TCC No. 65 Proposta Metodológica para Capacitação de Recursos Humanos para a Gestão Participativa de Bacias Hidrográficas: Estudo de

Autor(a): Udson Dias de Oliveira Júnior

Caso Comitê Camboriú - SC

Orientador(a): Dr Marcus Polette; Banca avaliadora: MSc Leonardo Rubi Rörig, Dr Luis Antonio O Proença

Data de Apresentação: $17 / 11 / 2000$

\begin{tabular}{l|l} 
TCC No. 66 & \\
\hline
\end{tabular}

Autor(a): Guilherme de Souza Soares

Orientador(a): MSc Humber Agrelli de Andrade; Banca avaliadora: Dr José Angel Alvares Perez, Dr Antonio Carlos Beaumord

Data de Apresentação: 23/11/2000

TCC No. 67 Inventário das Autuações Realizadas pela Polícia de Proteção Ambiental na Zona Costeira do Estado de Santa Catarina

Autor(a): Anahi Ribeiro

Orientador(a): Dr Marcus Polette; Banca avaliadora: Oc. Alexandre Mazzer, Tenente Antônio João de Mello Junior

Data de Apresentação: 27/11/2000

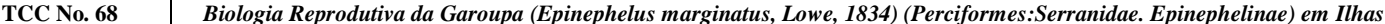

Autor(a): Äthila Bertoncini Andrade

Orientador(a): MSc Maurício Hostim Silva; Banca avaliadora: MSc Roberto Warlich, MSc Marcelo Rodrigues Ribeiro

Data de Apresentação: 23/11/2000

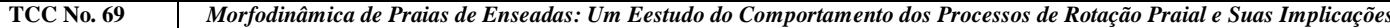
Ambientais

Autor(a): Lindino Benedet Filho

Orientador(a): MSc Antonio Henrique da Fontoura Klein; Banca avaliadora: Dr. Elirio Toldo Jr, MSc Delamar Shumacher

Data de Apresentacão: $24 / 11 / 2000$

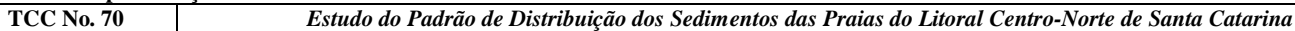

Autor(a): Graziela Miot da Silva

Orientador(a): MSc Antonio Henrique da Fontoura Klein; Banca avaliadora: Dr Elirio Toldo Jr, MSc Fernando Luiz Diehl

Data de Apresentação: 24/11/2000

\begin{tabular}{l|l} 
TCC No. 71 & \\
\hline Autor(a): Marcus Adonai Castro da Silva
\end{tabular}

Orientador(a): MSc Marco Antônio Bacellar Barreiros; Banca avaliadora: MSc Kátia Naomi Kuroshima, MSc. Gilberto Caetano Manzoni

Data de Apresentação: 24/11/2000

TCC No. 72 Caracterização Estrutural do Ecossistema Manguezal no Ribeirão Cassino da Lagoa, Praia Brava, Itajaí

Autor(a): Márcio Fabiano de Souza

Orientador(a): Dra Mônica Maria Pereira Tognella De Rosa; Banca avaliadora: MSc. Simone Rabelo da Cunha, Dr. Marcus Polette

Data de Apresentação: $24 / 11 / 2000$

TCC No. 73 Descrição e Dinâmica da frota de traineiras no Porto de Itajaí, SC, entre 1997 e 1999

Autor(a): Daniela Sarcinelli Occhialini

Orientador(a): C Paulo Ricardo Schwingel; Banca avaliadora: MSc. Humber Agrelli de Andrade, MSc. Roberto Wahrlich

Data de Apresentação: 27/11/2000

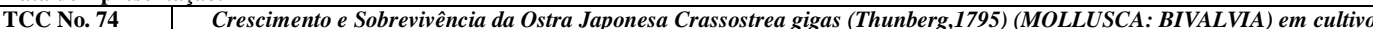

Autor(a): Gláucio Vintém flutuante (Balsa) na Enseada de Porto Belo - SC

Orientador(a): MSc. Gilberto Caetano Manzoni; Banca avaliadora: MSc Adriano Weidner Cacciatori Marenzi, MSc Luiz Eduardo Carvalho Bonilha

Data de Apresentação: 06/07/2001

TCC No. 75 Sistema de Dunas Costeiras da Praia de Navegantes - Gravatá (SC): Morfodinâmica, Usos Riscos e Gestão.

Autor(a): Paola Fracasso

Orientador(a): MSc. Fernando Luiz Diehl; Banca avaliadora: MSc Antonio H F klein, MSc Delamar Schumaker 
Anexo 1 - Continuação.

\begin{tabular}{|l|l} 
TCC No. 76 & Avaliação das características físicas, químicas e microbiológicas da enseada de Camboriú-SC
\end{tabular}

Autor(a): Caroline Fontelles Ternes

Orientador(a): MSc. Kátia Naomi Kuroshima; Banca avaliadora: Dr Marco Antônio Bacellar Barreiros, MSc Janete Feijó

Data de Apresentação: 11/07/2001

\begin{tabular}{l|ll} 
TCC No. 77 & Efeito da Temperatura, da Salinidade e do Chumbo sobre o Mecanismo de resistência a Multixenobióticos (MXR) no Mexilhão
\end{tabular}

Autor(a): Juliana Kuehn

Orientador(a): MSc. Marcos Luiz Pessatti; Banca avaliadora: Dr José Domingos Fontana, MSc Charrid Resgala Jr

Data de Apresentação: 11/07/2001

TCC No. 78 Variações das taxas Fisiológicas sob Condições Crônicas para o Mexilhão Perna perna

Autor(a): Elisangela de Souza Brasil

Orientador(a): MSc. Charrid Resgalla Júnior; Banca avaliadora: MSc Luiz Eduardo Bonilha, MSc Macos Pessati

Data de Apresentação: $12 / 07 / 2001$

TCC No. 79 Evolução das Taxas de Assoreamento ao Longo do Estuário do Rio Itajai-açu, através da Análise Multitemporal de Cartas

Autor(a): Vinicius Barbosa do Carmo Batimétricas

Orientador(a): Dr. Francisco Caruso Gomes Júnior; Banca avaliadora: Eng Luis Antonio Braga Martins, MSc Carlos Augusto schettini

Data de Apresentação: 10/07/2001

TCC No. 80 Avaliação da Composição Química do Rejeito Produzido pelas Indústrias que Processam Pescado na Região de Itajaí e

Autor(a): Fábio Luiz Ferreira Bruschi Navegantes, Santa Catarina

Orientador(a): MSc. Marcos Luiz Pessatti; Banca avaliadora: Dr Francisco Carlos Deschamps, MSc Tania de Lima Pacheco Pessatti

Data de Apresentação: 12/07/2001

TCC No. 81 Teste de Substituição Parcial de Farinha de Peixe pela farinha de Víscera de Atum, como fonte de Proteína na Ração par TilápiaAutor(a): Luís Fernando Valmorbida

Orientador(a): Dr.. Gosuke Sato; Banca avaliadora: Dr Hilton Amaral, MSc Adriano Marenzi

Data de Apresentação: $16 / 07 / 2001$

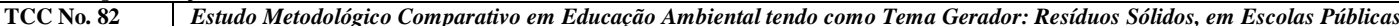
Minucipais de Itajaí -SC

Autor(a): Irene Marschalek

Orientador(a): MSc. Luiz Eduardo Carvalho Bonilha; Banca avaliadora: Dr Yára Christina Cezário, Oc José Matarezi

Data de Apresentação: $13 / 07 / 2001$

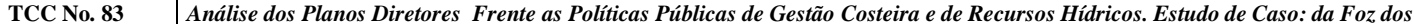

Autor(a): Diana Carla Floriani

Orientador(a): Dr. Marcus Polette; Banca avaliadora: Dr Alexandre Lerípio, Dra. Isa de Oliveira Rocha

Data de Apresentação: $10 / 07 / 2001$

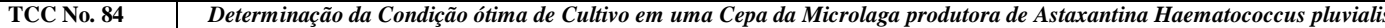
\begin{tabular}{l} 
(Chlorophyceae) \\
\hline
\end{tabular}

Autor(a): Rodrigo Cavalheiro

Orientador(a): MSc. Leonardo Rubi Rörig; Banca avaliadora: Dr Luis Antonio O Proença, Dr José Domingos Fontana

Data de Apresentação: $11 / 07 / 2001$

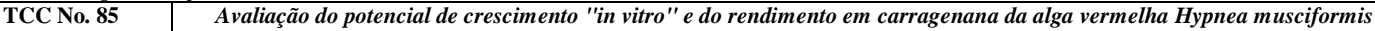

Autor(a): Gláucia Bezerra de Lima (Hypneaceae, Rhodophyta)

Orientador(a): Dr. Simone Rabelo da Cunha; Banca avaliadora: Dra. Zenilda Bouzon, MSc Adriano Weidner Cacciatori Marenzi

Data de Apresentação: 22/11/2001

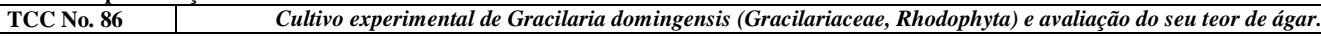

Autor(a): Fernando Dias Pazeto

Orientador(a): Dr. Simone Rabelo da Cunha; Banca avaliadora: Dra. Zenilda Bouzon, MSc Adriano Weidner Cacciatori Marenzi

Data de Apresentação: $22 / 11 / 2001$

\begin{tabular}{|l|l} 
TCC No. 87 & Comparacão entre os métodos espanhol e francês de cultivo de mexilhões
\end{tabular}

Autor(a): Marco Antônio Coelho de Souza Filho

Orientador(a): Msc. Adriano Weidner Cacciatori Marenzi; Banca avaliadora: Dr. Hilton Amaral, MSc. Luis Eduardo Bonilha

Data de Apresentação: $21 / 11 / 2001$

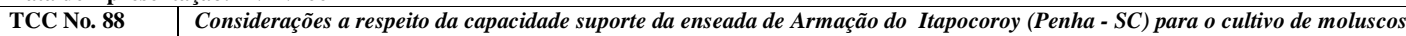
marinhos

Autor(a): Inaê Adorno de Araújo

Orientador(a): Msc. Luiz Eduardo C. Bonilha; Banca avaliadora: Dr. Luis A. de O Proença, MSc. Charrid Resgalla ,Jr. Oc. Rodrigo Medeiros

Data de Apresentação: 21/11/2001

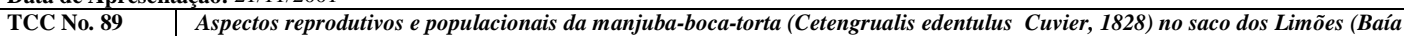
Sul, Florianópolis)

Autor(a): José Maria de Souza da Conceição

Orientador(a): Msc.. Marcelo Rodrigues Ribeiro; Banca avaliadora: Dr. Paulo Ricardo Schwingel, Dra Maria Paula Mellito da Silveira

Data de Apresentação: $21 / 11 / 2001$

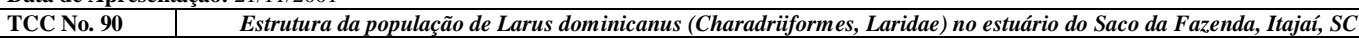

Autor(a): Luís Augusto Ebert

Orientador(a): Dr. Joaquim Olinto Branco; Banca avaliadora: Dr. Tito Cesar Marcos de Almeida, Dra Maria José Lunardon-Branco 
PROENÇA et al.: Projeto de graduação e a produção científica: Oceanografia CTTMar/UNIVALI.

Anexo 1 - Continuação.

\begin{tabular}{|c|c|}
\hline TCC No. 91 & Avaliação da toxicidade do extrato hidrossolúvel de Heterosigma akashiwo (rafidoficea) Hada 1968 \\
\hline \multicolumn{2}{|c|}{$\begin{array}{l}\text { Autor(a): Márcio da Silva Tamanaha } \\
\text { Orientador(a): Dr. Luís Antônio de Oliveira Proença; Banca avaliadora: Dr. Adair R.S.Santos, MSc MSc. Marcos Luiz Pessatti, MSc. Charrid } \\
\text { Resgalla Jr. } \\
\text { Data de Apresentação: 23/11/2001 }\end{array}$} \\
\hline TCC No. 92 & $\begin{array}{r}\text { Tômbolos, formas cuspadas e saliências na linha de costa do litoral de Santa Catarina: descrição das formas, análise das relações } \\
\text { morfológicas apresentadas pelas feições e suas implicaçôes ambientais }\end{array}$ \\
\hline \multicolumn{2}{|c|}{$\begin{array}{l}\text { Autor(a): Narbal Andriani Júnior } \\
\text { Orientador(a): MSc. Antonio Henrique da Fontoura Klein; Banca avaliadora: Dra. Patricia Faria, Dr. Moyses Tessler } \\
\text { Data de Apresentação: } 21 / 11 / 2001\end{array}$} \\
\hline TCC No. 93 & $\begin{array}{c}\text { Comparação entre parâmetros de onda estimados no domínio do tempo e dominio da frequência para o litoral sul de Arraial do } \\
\text { Cabo }-R J\end{array}$ \\
\hline \multicolumn{2}{|c|}{$\begin{array}{l}\text { Autor(a): Rafael Bonanata da Rocha } \\
\text { Orientador(a): MSc.. João Luiz Baptista de Carvalho; Banca avaliadora: Dra. Patricia Faria, Dr. José Henrique Alves } \\
\text { Data de Apresentação: 22/11/2001 }\end{array}$} \\
\hline TCC No. 94 & 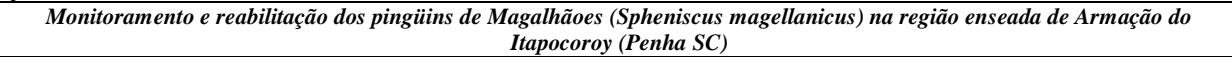 \\
\hline \multicolumn{2}{|c|}{$\begin{array}{l}\text { Autor(a): Erich Cerchiari } \\
\text { Orientador(a): MSc.. Gilberto Caetano Manzoni; Banca avaliadora: Dr. Joaquim Olinto Branco, Dr. Andre Barreto } \\
\text { Data de Apresentação: } 20 / 11 / 2001\end{array}$} \\
\hline TCC No. 95 & Efetividade de recersão sexual em três tamanhos de tilápia do Nilo (Oreochromis niloticus) \\
\hline \multicolumn{2}{|c|}{$\begin{array}{l}\text { Autor(a): Giuliano Palemao Carlos Maia Huergo } \\
\text { Orientador(a): Dr. Gosuke Sato; Banca avaliadora: Dr Hilton Amaral , MSc. Gilberto Caetano Manzoni } \\
\text { Data de Apresentação: } 22 / 11 / 2001\end{array}$} \\
\hline TCC No. 96 & $\begin{array}{c}\text { Análise prelimnar do uso de imagem satélite na determinação da batimetria em águas costeiras. Um exemplo dc } \\
\text { Conceição }\end{array}$ \\
\hline \multicolumn{2}{|c|}{$\begin{array}{l}\text { Autor(a): Mônica Marrani Stoelzle } \\
\text { Orientador(a): Dr. Oldemar de Oliveira Carvalho Júnior; Banca avaliadora: Dr Rosana Maria Rodrigues, Dr. Eduardo Soriano-Sierra } \\
\text { Data de Apresentação: } 23 / 11 / 2001\end{array}$} \\
\hline TCC No. 97 & Biomassa e fatores causais das florações de Euglenofíceas psâmicas na praia de Balneário Camboriú - SC \\
\hline \multicolumn{2}{|c|}{$\begin{array}{l}\text { Autor(a): César Bacila Munhoz da Rocha } \\
\text { Orientador(a): MSc. Leonardo Rubi Rörig; Banca avaliadora: Dr Marinês Garcia, MSc. Jurandir Pereira Fo. } \\
\text { Data de Apresentação: 22/11/2001 }\end{array}$} \\
\hline TCC No. 98 & Bases para o manejo de Sargassum cymosum (Phaeophyta - Fucales) na Enseada de Armação do Itapocoroy, Penha, SC \\
\hline \multicolumn{2}{|c|}{$\begin{array}{l}\text { Autor(a): Luiz Laureno Mafra Júnior } \\
\text { Orientador(a): Dr. Simone Rabelo da Cunha; Banca avaliadora: Dr Dr Paulo Horta, MSc. Adriano Weidner Cacciatori Marenzi } \\
\text { Data de Apresentação: 22/11/2001 }\end{array}$} \\
\hline TCC No. 99 & $\begin{array}{l}\text { Usos, costumes e tecnologias associados a "nogeuira" (Aleurites moluccana - Euphorbiaceae). O "saber fa } \\
\text { suporte para o desenvolvimento sustentável e a educação ambiental comunitária no município de Bomb }\end{array}$ \\
\hline \multicolumn{2}{|c|}{$\begin{array}{l}\text { Autor(a): Fernando Fiedler } \\
\text { Orientador(a): Oc José Matarezi ;Banca avaliadora: MSc. Cristiane Manique Barreto , MSc. Luiz Edurado de Carvalho Bonilha } \\
\text { Data de Apresentação: 23/11/2001 }\end{array}$} \\
\hline TCC No. 100 & $\begin{array}{c}\text { Determinação da idade e crescimento da cavalinha (Scomber japonicus Houttuyn, 1782), desembarcada pela froto } \\
\text { traineiras no porto pesqueiro de Itajaí/Navegantes }\end{array}$ \\
\hline \multicolumn{2}{|c|}{$\begin{array}{l}\text { Autor(a): Alexandre Piccoli Grando } \\
\text { Orientador(a): MSc. Marcelo Rodrigues Ribeiro; Banca avaliadora: Dr José Angel Alvarez Perez, MSc. Humber Agrelli de Andrade } \\
\text { Data de Apresentação: 23/11/2001 }\end{array}$} \\
\hline TCC No. 101 & Análise de tamques de contato voltados para a educação ambiental em áreas costeiras e unidades de conse \\
\hline \multicolumn{2}{|c|}{$\begin{array}{l}\text { Autor(a): Fabrício Menezes Ramos } \\
\text { Orientador(a): Oc. José Matarezi; Banca avaliadora: Dra Yára Cristina Cezário, MSc. Ricardo Corbetta } \\
\text { Data de Apresentação: } 23 / 11 / 2001\end{array}$} \\
\hline TCC No. 102 & Geologia e uso das terras da Praia do Estaleiro - SC e algumas considerações sobre seus aspectos ambientais. \\
\hline \multicolumn{2}{|c|}{$\begin{array}{l}\text { Autor(a): Rafael Mueller Petermann } \\
\text { Orientador(a): MSc. Sérgio Freitas Borges; Banca avaliadora: MSc. Seegey Alex Araújo, MSc. Maria Inês dos Santos } \\
\text { Data de Apresentação: } 09 / 07 / 2002\end{array}$} \\
\hline TCC No. 103 & Carcinofauna acompanhante na pesca artesanal do camarão sete-barbas na Armação do Itap \\
\hline \multicolumn{2}{|c|}{$\begin{array}{l}\text { Autor(a): Hélio Augusto Alves Fracasso } \\
\text { Orientador(a): Dr. Joaquim Olinto Branco; Banca avaliadora: MSc. Gilberto Caetano Manzoni, MSc. Roberto Wahrlich } \\
\text { Data de Apresentação: 09/07/2002 }\end{array}$} \\
\hline TCC No. 104 & Produção de juvenil de Tilápia-do-Nilo (Oreochromis niloticus) no período de outono / inverno, et \\
\hline \multicolumn{2}{|c|}{$\begin{array}{l}\text { Autor(a): Bruno Kupsch } \\
\text { Orientador(a): Dr. Hilton Amaral Júnior; Banca avaliadora: Dr. Gosuke Sato, MSc. Gilberto C. Manzoni } \\
\text { Data de Apresentação: } 09 / 07 / 2002\end{array}$} \\
\hline TCC No. 105 & Abundância e aspectos da reprodução de Larus dominicanus (Charadriiforfe, Laridae) no Arquipélago do Arvoredo, SC. \\
\hline utor(a): Hede & $\begin{array}{l}\text { siano Moritz Júnior } \\
\text { oaquim Olinto Bran } \\
\text { âo: } 09 / 07 / 2002\end{array}$ \\
\hline
\end{tabular}


Anexo 1 - Continuação.

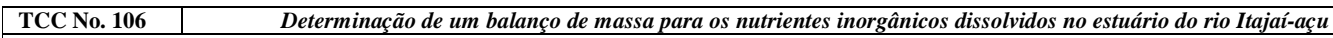
Autor(a): Luciana de Carvalho Spillere

Orientador(a): MSc. Jurandir Pereira Filho; Banca avaliadora: MSc Leonardo Rubi Rorig, MSc. Katia Naomi Kuroshima

Data de Apresentação: 27/06/2002

TCC No. 107 Estudo do emprego de motor na pesca do gerival na Baía da Babitonga - SC

Autor(a): Alessandro Agno Maia Pacheco

Orientador(a): MSc. Roberto Wahrlich; Banca avaliadora: MSc Marco Aurelio Bailon, MSc. Ana Maria Torres Rodrigues

Data de Apresentação: 10/07/2002

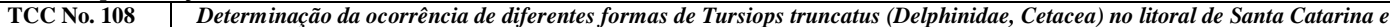
norte do Rio Grande do Sul

Autor(a): Cristina Maschio de Miranda

Orientador(a): Dr. André Barreto;Banca avaliadora: Dr. Maurício Hostim Silva, Dr. Paulo Ricardo Pezzuto

Data de Apresentação: 10/07/2002

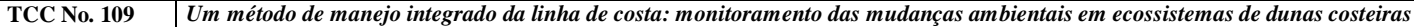

Autor(a): Fabrício Gabriel Mora

Orientador(a): MSc. Fernando Luiz Diehl; Banca avaliadora: MSc. Antonio Henrique da Fontoura Klein, Dr Elírio Ernestino Toldo Jr.

Data de Apresentação: 15/07/2002

\begin{tabular}{l|l} 
TCC No. 110 & Idade, crescimento e ciclo reprodutivo da sardinha-lage (Opisthonema oglinum) para o sudeste e sul do Brasil.
\end{tabular}

Autor(a): Marcelo Costa Feltrin

Orientador(a): Dr. Paulo Ricardo Schwingel; Banca avaliadora: MSc. Humber A. Andrade, Dr. José Angel Perez

Data de Apresentação: $11 / 07 / 2002$

TCC No. 111

Autor(a): José Renato Derntl

Orientador(a): MSc. Antonio Henrique da Fontoura Klein; Co-Orientador: Dr Elírio Ernestino Toldo Jr.

Banca avaliadora: MSc. João Thadeu de Menezes, MSc. José Gustavo N. de Abreu Dr Elírio Ernestino Toldo Jr.

Data de Apresentação: 15/07/2002

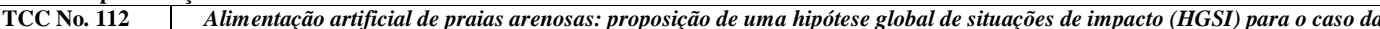

Autor(a): Emílio Marcelo Dolichney praia central de Balneário Camboriú, SC.

Orientador(a): Dr. Antonio Carlos Beumord; Banca avaliadora: MSc. Leo Lynce Valle de Lacerda, MSc. Fernando Luiz Diehl

Data de Apresentação: $16 / 07 / 2002$

TCC No. $113 \quad$ Utilização da microestrutura do estatólito para a determinação da idade e estimativas de taxas de crescimento da lula Loligo plei Autor(a): Daniela Cordella Aguiar

Orientador(a): Dr. José Angel Alvarez Perez; Banca avaliadora: Dr. Paulo Ricardo Pezzuto, MSc. Humber Andrade

Data de Apresentação: $11 / 07 / 2002$

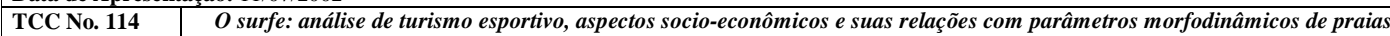
arenosas oceânicas. Caso de estudo praia Brava, Itajai, $S C$

Autor(a): Gustavo Dafferner

Orientador(a): Dr. Guilherme Guimarães Santana; Co-Orientador: MSc. Antonio Henrique da Fontoura Klein

Banca avaliadora: Dr. Luis A O Proença, MSc. Rodrigo Medeiros, MSc. Antonio Henrique da Fontoura Klein

Data de Apresentação: $15 / 07 / 2002$

TCC No. 115 Ecologia trófica da sardinha-verdadeira (na costa sudeste-sul do Brasil

Autor(a): Fabíola Schneider

Orientador(a): Dr. Paulo Ricardo Schwingel; Banca avaliadora: Dr. Paulo Ricardo Pezzuto, MSc. Marcelo Rodriguez Ribeiro

Data de Apresentação: 11/07/2002

\begin{tabular}{|l|l}
\hline TCC No. 116 & \\
\hline Autor(a): Gabiela Faria Oliveira
\end{tabular}

Composição dos ácidos graxos da fração lipídica de resíduos industriais da pesca

Autor(a): Gabriela Faria Oliveira

Orientador(a): MSc. Marcos Luiz Pessatti; Banca avaliadora: Dr. Francisco Carlos Deschamps, Dr. Marco Antonio Barreiros

Data de Apresentação: 12/07/2002

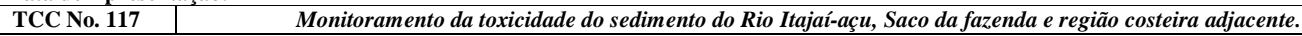

Autor(a): Rafaela Michels da Silveira

Orientador(a): MSc. Charrid Resgalla Júnior; Banca avaliadora: MSc Kátia N. Kuroshima, Dr. Carlos. A.F. Schettini

Data de Apresentação: $12 / 07 / 2002$

TCC No. 118 Programa de Monitoramento Ambiental Voluntário nas escolas: Uma experiência piloto em educação ambiental "Clube Olho Vivo".

Autor(a): Tatiane Mentges

Orientador(a): MSc. Luiz Eduardo Carvalho Bonilha; Co-Orientador: Oc. José Matarezi

Banca avaliadora: Msc. Cleuza Guaita Peralta, Oc. José Matarezi

Data de Apresentação: $10 / 07 / 2002$

TCC No. $119 \quad$ Aspectos bioecológicos dos peixes associados a maricultura na Ilha de Porto Belo, em Porto Belo, Santa Catarina Brasil

Autor(a): Vicente Nagib Duarte Figna

Orientador(a): Dr. Maurício Hostim Silva; Banca avaliadora: MSc Adriano Weidner Cacciatori Marenzi, MSc. Luiz Edurado de Carvalho Bonilha

Data de Apresentação: 12/07/2002 